Revue des patrimoines

$41 \mid 2019$

Patrimoines gastronomiques. Définitions, typologies et enjeux de conservation

\title{
La faïencerie de Lunéville. Du repérage d'objets à la patrimonialisation participative
}

The Lunéville faience factory, identifying objects and participating in their heritage designation

Jeannine Guenot et Denis Saillard

\section{(2) OpenEdition}

Journals

Édition électronique

URL : http://journals.openedition.org/insitu/26079

DOI : $10.4000 /$ insitu. 26079

ISSN : $1630-7305$

Éditeur

Ministère de la Culture

Référence électronique

Jeannine Guenot et Denis Saillard, « La faïencerie de Lunéville. Du repérage d'objets à la

patrimonialisation participative », In Situ [En ligne], 41 | 2019, mis en ligne le 09 décembre 2019, consulté le 19 décembre 2019. URL : http://journals.openedition.org/insitu/26079; DOI : 10.4000/ insitu.26079

Ce document a été généré automatiquement le 19 décembre 2019.

\section{cc) (†)}

In Situ Revues des patrimoines est mis à disposition selon les termes de la licence Creative Commons Attribution - Pas d'Utilisation Commerciale - Pas de Modification 4.0 International. 


\section{La faïencerie de Lunéville. Du repérage d'objets à la patrimonialisation participative}

The Lunéville faience factory, identifying objects and participating in their heritage designation

Jeannine Guenot et Denis Saillard

1 L'association des Amis de la faïence ancienne de Lunéville Saint-Clément ${ }^{1}$, fondée en 1998 par maitre Pierre Poncet et quelques passionnés de faïence, compte un peu plus d'une centaine d'adhérents, français et étrangers. Association loi 1901, elle poursuit deux objectifs essentiels : étudier et faire connaître le patrimoine faïencier des entreprises célèbres ou méconnues de Lunéville (Meurthe-et-Moselle) et sa région (Badonviller, Pexonne, Domèvre, etc.), et partager les connaissances acquises avec les amateurs, étudiants, chercheurs et professionnels du patrimoine.

Dans cette perspective, l'association organise régulièrement des expositions, dont le thème varie en fonction des travaux de recherche menés, et publie des ouvrages, dont les catalogues de ces expositions. Une revue, Réverbère, et un organe de liaison, Le Journal des Amis, diffusent et promeuvent les activités culturelles et scientifiques, en collaboration avec les institutions patrimoniales et les chercheurs académiques, et les actions de valorisation (salons). Le public des jeunes écoliers, des lycéens et des étudiants est particulièrement pris en compte, par exemple dans les visites guidées et conférences organisées pour valoriser les collections de la salle d'exposition permanente qu'administre l'association près du site de l'ancienne faïencerie de Lunéville.

3 Par leurs multiples implications, les Amis de la faïence ancienne de Lunéville SaintClément apportent leur contribution à l'histoire matérielle, dont les apports ne sont plus à démontrer². De même que des études successives ont bien montré l'évolution de l'usage des couverts, comme l'introduction, très progressive, de la fourchette en France 
aux XVI ${ }^{\mathrm{e}}$-XVII ${ }^{\mathrm{e}}$ siècles, l'histoire des services de table au fil des siècles a fait l'objet de recherches significatives de la part des historiens du patrimoine lié à l'alimentation.

La présente étude de cas, en forme de témoignage consacré au secteur des faïences, propose de rendre compte des formes de médiation mises actuellement en œuvre par l'association. Elle répond à la démarche participative actuelle dans la mesure où elle invite toute personne possédant des pièces de faïence à contribuer à une recherche documentaire et à des expositions.

\section{Les faïences dans l'histoire de la gastronomie}

5 Se développant dès la fin du Moyen Âge et durant la Renaissance (Lyon, Nevers...), la faïence de table connaît en France un essor plus marqué au cours des $\mathrm{XVII}^{\mathrm{e}}$ et XVIII siècles ${ }^{3}$. La cour royale et l'aristocratie elles-mêmes ont adopté son usage en dépit de sa fragilité et de son prestige moindre par rapport aux services en métal (étain, argent, voire or). C'est ce qu'attestent les témoignages de la Princesse Palatine et du duc de Saint-Simon sur les mutations consécutives aux graves crises économiques survenues à la fin du règne de Louis XIV : en 1709, à la Cour, «tout ce qu'il y eut de considérable se mit en huit jours en faïence "; le duc d'Antin vint "à Paris choisir force porcelaine admirable, qu'il eut à grand marché, et enlever deux boutiques de faïence qu'il fit porter pompeusement à Versailles ${ }^{4}$.

6 Les services de table en faïence devinrent de plus en plus sophistiqués et leur décor recherché. Les techniques de fabrication se sont diversifiées et les ateliers se sont multipliés en France et en Europe ${ }^{5}$. À la fin du XVIII ${ }^{e}$ siècle, la solidité des pièces des nouvelles fabriques (Gien, Lunéville, Choisy-le-Roi...) est sans commune mesure avec celle des époques précédentes. Les familles des milieux populaires, elles, disposent de quelques pièces de vaisselle en faïence blanche, qui complètent celles en bois. La démocratisation des services de faïence ornée n'intervient que lentement. Elle s'accélère dans la seconde moitié du XIX ${ }^{e}$ siècle grâce à de nouvelles techniques, qui entraînent l'augmentation spectaculaire de la production et l'abaissement de son coût, tandis que le niveau de vie moyen s'élève. Par la suite, jusqu'à la concurrence récente d'autres matériaux et au développement d'autres pratiques sociales, un très grand nombre de familles françaises en possède.

7 L'histoire culturelle s'intéresse désormais aussi de près à ces objets ${ }^{6}$, ne serait-ce qu'en raison de leur rôle de médiation par le truchement de leur ornementation: motifs floraux, scènes de chasse... ou encore récit politique ou historique ${ }^{7}$.

\section{Quelques jalons de l'histoire des faïenceries de Lunéville}

8 De nos jours, à Lunéville (Meurthe-et-Moselle), le quartier de la faïencerie a été transformé en zone d'activités. Il ne reste que quelques bâtiments restaurés dans la seconde moitié $\mathrm{du} \mathrm{xx}^{\mathrm{e}}$ siècle, après la fermeture du site. Pourtant, la mémoire faïencière est très vive dans la cité; de nombreux témoins (ouvriers, cadres...) ont connu l'entreprise en activité. 
9 Le site de Lunéville eut sa faïencerie dès 1730. Dirigée par Jacques Chambrette, la fabrique se situait sur le « chemin de Viller » tant que l'établissement fut en activité. En 1758, la famille Chambrette acquit un terrain à Saint-Clément, à $7 \mathrm{~km}$ environ, pour des raisons douanières: Lunéville était l'une des cités les plus importantes de Lorraine grâce à la présence du château ducal, alors que le village de Saint-Clément appartenait à l'évêque de Metz. À la mort de Chambrette, ses héritiers ne tardèrent pas à se séparer et les deux entreprises ainsi constituées fonctionnèrent parallèlement de 1763 à 1892.

La manufacture de Lunéville fut soutenue dès l'origine par Élisabeth-Charlotte d'Orléans, duchesse de Lorraine, épouse du duc Léopold I Ir puis régente de 1729 à 1737, qui lui accorda des exemptions fiscales. Stanislas Leszczynski, dernier duc de Lorraine et de Bar (1737-1766), par deux fois roi de Pologne (1704-1709 puis 1733-1736), éleva la manufacture au rang de manufacture royale ${ }^{8}$. À partir de 1786, une nouvelle dynastie de faïenciers, les Keller, prit possession de la manufacture; à la suite d'un mariage, l'entreprise associa le nom de Keller à celui de Guérin. Les deux sites furent réunifiés en 1892.

11 En 1922, la faïencerie passa aux mains de la famille Fenal. Les guerres successives et la concurrence de nouveaux matériaux engendrèrent son déclin. Un incendie, en 1968, diminua la production à Lunéville. De nos jours, seul subsiste le site de Saint-Clément, qui fonctionne avec très peu de salariés. La menace d'une fermeture définitive n'est pas écartée.

12 Cet héritage mérite l'attention car les productions lunévilloises sont de très belle qualité en raison des pâtes et des couleurs employées. Elles possèdent une notoriété nationale et internationale en dépit de l'existence de très nombreux autres foyers de production en France et en Europe. La Belle Époque et l'entre-deux-guerres furent une période faste pour l'entreprise, qui se lança alors dans de nombreuses innovations techniques et artistiques. C'est vers 1900 que la faïencerie fut la plus prospère : elle employait près de 1300 ouvriers et ses productions connaissaient un grand succès commercial.

Les objets étudiés ici ont été fabriqués fin $\mathrm{XIX}^{\mathrm{e}}$-début $\mathrm{xx} \mathrm{x}^{\mathrm{e}}$ siècle, période à laquelle la fabrique atteignit son apogée grâce à des artistes et des ouvriers particulièrement imaginatifs (Edmond Lachenal, Louis Majorelle, Géo Condé...), qui multiplièrent les formes et contribuèrent au renouvellement de la conception de l'art faïencier. Si la production lunévilloise n'est pas entièrement consacrée aux services de table, ces derniers en représentent une part très importante.

\section{La réunion d'objets pour l'exposition « Rovina » en 2017}

L'association des Amis de la faïence ancienne de Lunéville Saint-Clément, fondée en $1998^{\circ}$, s'emploie à préserver et à faire connaître ce patrimoine par le biais de publications et d'expositions. Aucune période n'est négligée. Souvent, des remarques de ses lecteurs et de ses visiteurs incitent ses responsables à entreprendre des recherches sur un sujet. Les échanges instaurés, grâce au site internet de l'association, avec de simples curieux, des étudiants ou des chercheurs exigent des réponses précises, pour lesquelles ses membres, chercheurs non professionnels, appliquent les méthodes de la recherche historique. 

est le résultat de ce type d'échanges permanents. Évoquons d'abord les faits qui ont préludé au projet. L'association pratique un petit commerce de brocante, alimenté par des dons d'objets consentis par ses adhérents ou des personnes extérieures. Nous recevons souvent des courriels ainsi libellés: «Nous avons trouvé dans la maison de nos parents des faïences signées Lunéville...» ou encore, émanant de personnes cherchant à identifier une marque méconnue. Un dialogue s'engage alors. Les responsables de l'association demandent systématiquement des photographies de l'objet, du décor et des marques éventuelles, éléments diffusés aux membres pour recueillir toute information éventuelle. Si nécessaire, les Amis de la faïence se mettent en relation avec d'autres associations, des antiquaires, des musées voire des chercheurs académiques.

Dans le cas du projet «Rovina », une antiquaire a pu élucider l'origine d'une pièce particulière (fig. 1) et la signification de sa signature, qui comprend une partie écrite et une sorte de bouquet cachant presque totalement la marque de Lunéville, pourtant reconnaissable à sa couronne et quelques lettres. La faïence a été produite à Lunéville, indication confirmée dans les livres de comptes par des commandes d'objets émaillés non décorés, exécutées par les Rovina, famille d'Épinal à la tête d'un magasin de faïences, porcelaines et cristaux à partir de 1860. Les initiales associées au nom « Rovina » ont conduit en outre à entamer une recherche sur cette famille.

Figure 1

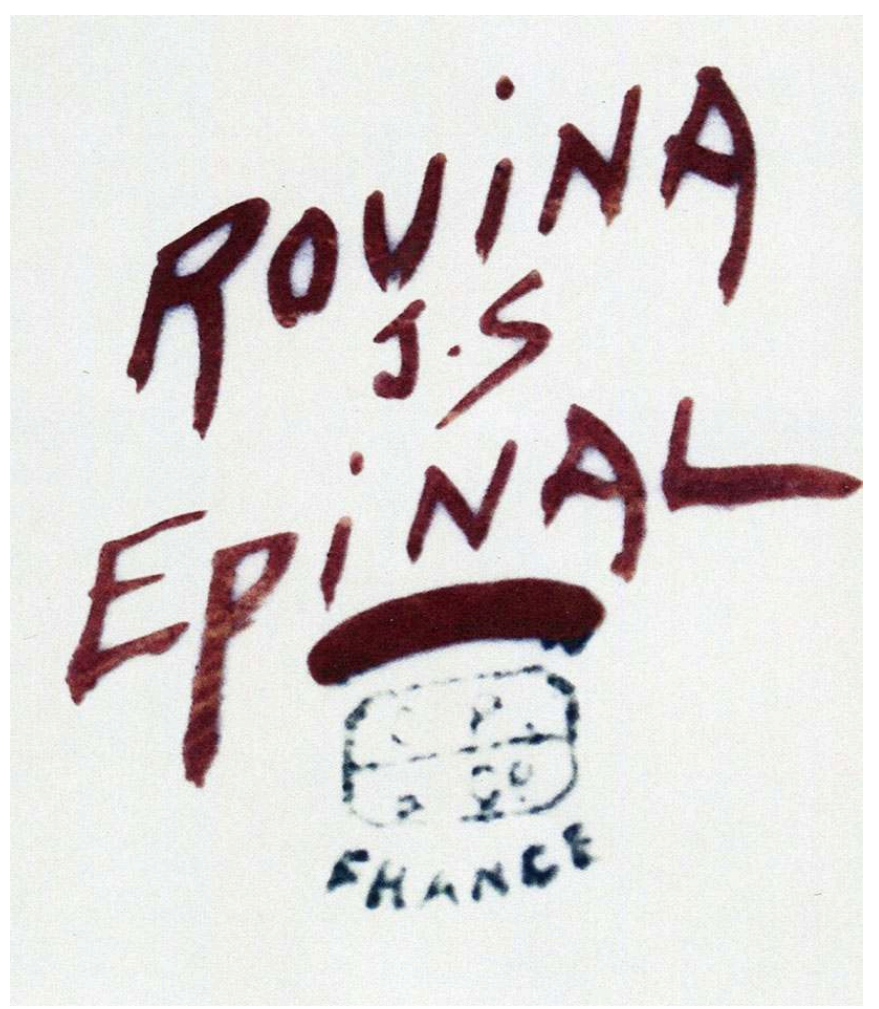

Signature « Rovina JS Épinal ».

(c) Association des Amis de la faïence ancienne de Lunéville Saint-Clément.

Les recherches généalogiques menées (fig. 2) ont permis d'attribuer correctement les initiales « JS » à l'époux de la petite-fille de la fondatrice de l'établissement Rovina, 
Jean-Pierre Schmitt. Chaque peintre de l'atelier devait garder la marque générale Rovina mais pouvait ajouter son propre nom. On a ainsi pu dater correctement les pièces et retracer une partie de leur histoire pour leur propriétaire.

Figure 2

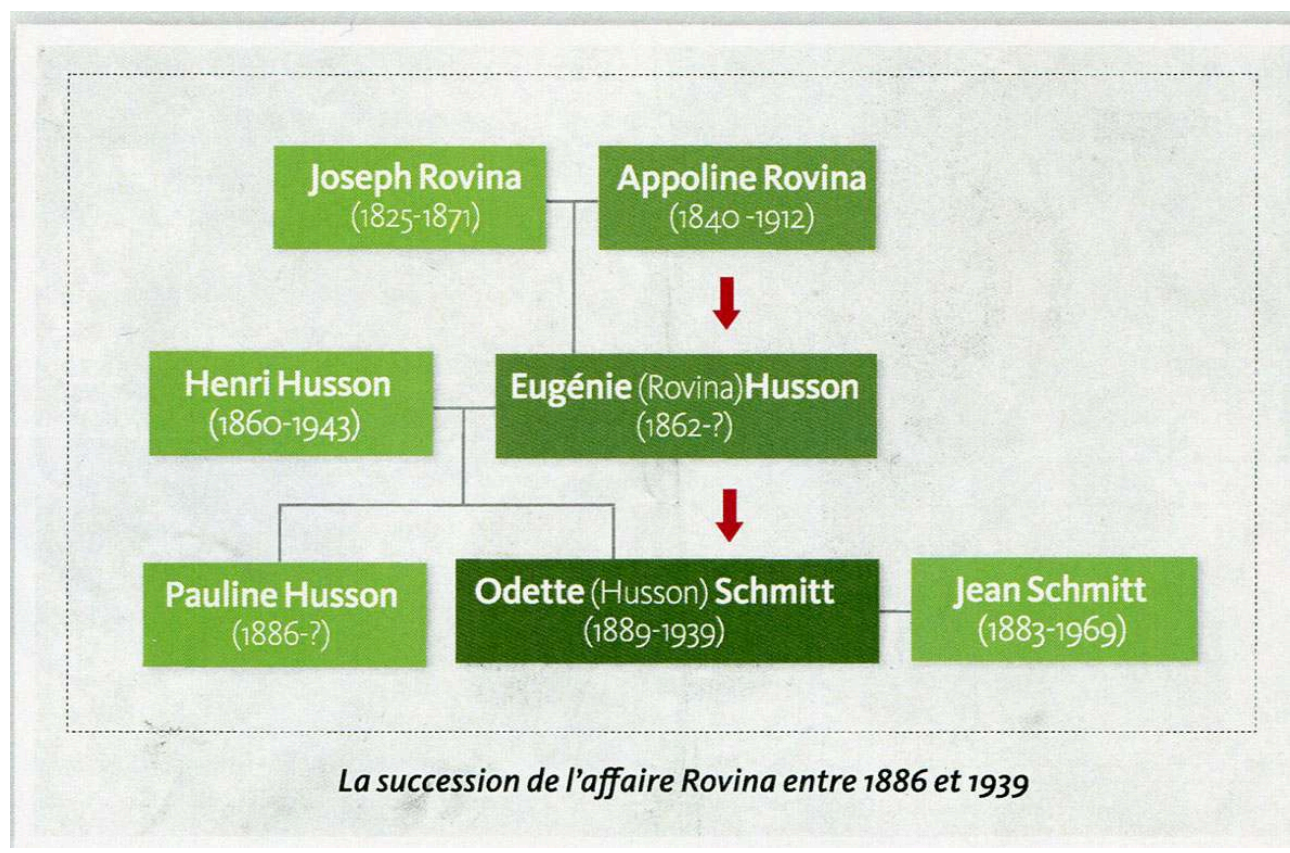

«Résumé de la filiation des Rovina ».

(c) Association des Amis de la faïence ancienne de Lunéville Saint-Clément.

Certains propriétaires recherchent, au-delà, dans leur histoire familiale, l'origine de la possession de cette pièce. Les témoignages reçus par l'association montrent que l'objet prend alors une valeur nouvelle et qu'il n'est plus question de le vendre, la recherche effectuée ayant fait naître l'intérêt. Quelques-uns vont jusqu'à rechercher d'autres pièces pour compléter leur service ou bien acquièrent des objets jugés plus originaux que des assiettes ou des tasses.

Pour les pièces "Rovina", ce début d'enquête a aussi piqué la curiosité des responsables de l'association: ils se sont mis en quête d'autres objets de même provenance et ont eu de nouveaux échanges avec l'antiquaire qui avait fourni les premières indications historiques. Mis en relation avec des clients qui lui avaient acheté des pièces de cet atelier spinalien, les membres de l'association ont pu photographier les pièces, qui leur ont aussi souvent été proposées en prêt pour d'éventuelles manifestations.

\section{Le temps de l'exposition}

Devant la quantité de pièces disponibles, l'association a dû opérer une sélection, fondée sur plusieurs critères: la représentativité des objets par rapport au propos de l'exposition, l'attractivité des pièces et leur capacité à susciter des questions et à placer le visiteur dans une situation d'interactivité. 
L'exposition « Rovina. Saga d'une famille de peintre sur faïence et porcelaine » s'est

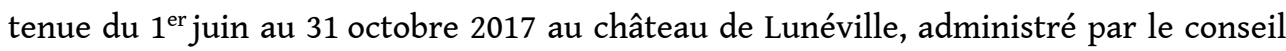
départemental de Meurthe-et-Moselle depuis l'incendie survenu en janvier 2003. Restauré partiellement, le monument attire de nombreux visiteurs, qui viennent de la région ou de l'étranger. Non exhaustive, l'exposition a été complétée par un catalogue ${ }^{10}$, destiné à diffuser les connaissances recueillies auprès du public, constitué d'amateurs, de chercheurs ou de professionnels. Ce rôle de passeur de savoirs sur l'art faïencier constitue l'activité la plus importante de l'association des Amis de la faïencerie ancienne de Lunéville Saint-Clément.

Une fois les pièces choisies, photographiées, décrites en détail, la scénographie devait soutenir la présentation du parcours de cette famille d'artistes et de ses collaborateurs. Plusieurs pôles ont été définis.

Le pôle chronologique présentait les productions des différents membres de la famille Rovina et l'évolution de la situation économique. Les signatures exposées ont ainsi permis de situer les lieux de vente ou les types de commandes (fig. 3, fig. 4). Le pôle sur les sources d'inspiration évoquait l'imitation des décors d'Aprey ${ }^{11}$, les créations personnelles ou les commandes particulières, comme le service à la cigogne, oiseau emblématique de l'aviateur René Fonck $^{12}$, créé spécialement pour lui. Un pôle particulier était dédié aux techniques, avec la présentation du petit matériel du peintre, du travail de l'or et du platine et de l'utilisation de la chromolithographie. Le dernier pôle, consacré aux différents peintres des ateliers Rovina ou sollicités par eux à la période la plus faste de l'entreprise, était consacré aux figures de Chasteloux, Résal, Balandier, Xélot ${ }^{13}$...

Figure 3

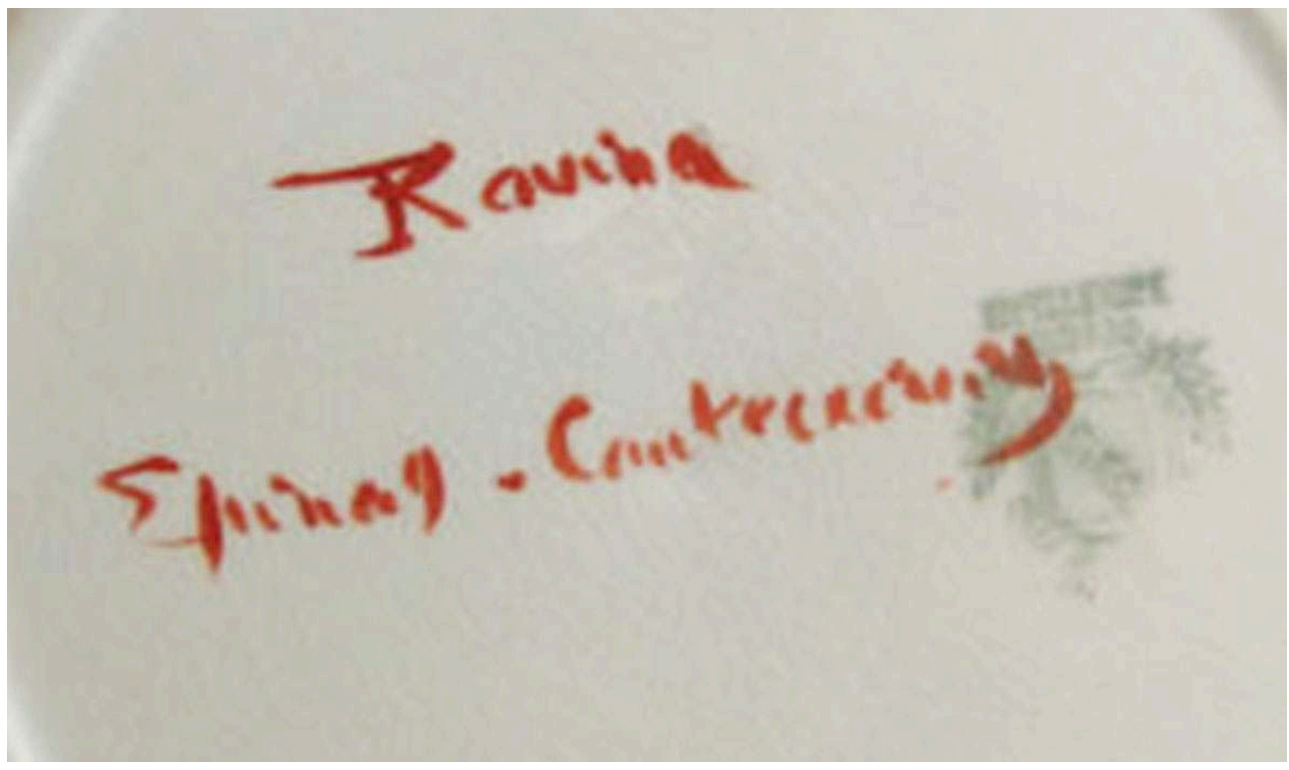

Signature «Rovina Épinal Contrexéville », indiquant les points de vente.

(c) Association des Amis de la faïence ancienne de Lunéville Saint-Clément. 
Figure 4

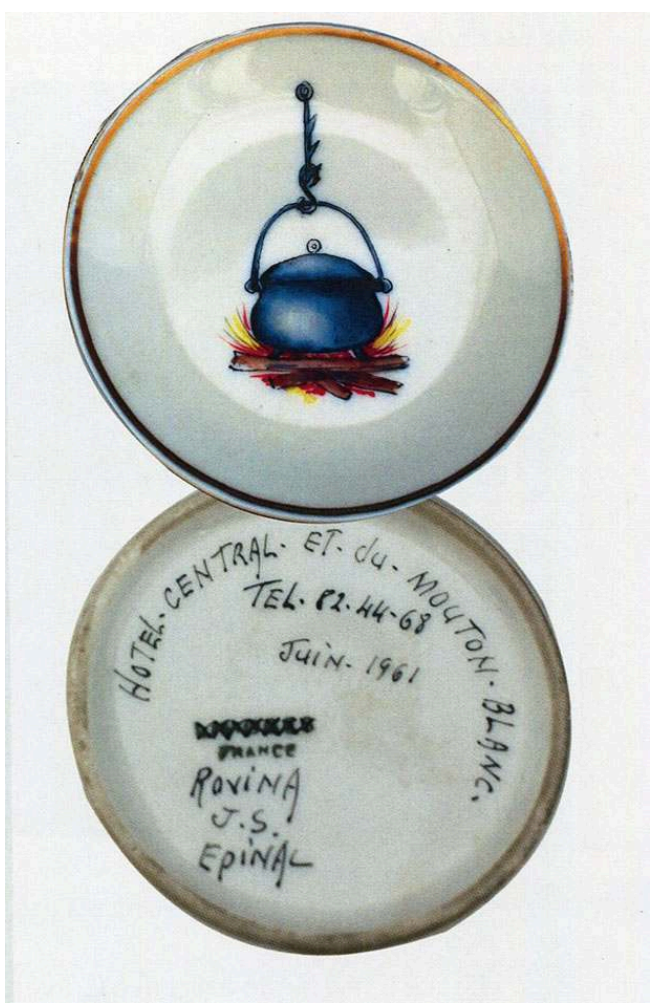

Article publicitaire ; petite coupelle offerte aux meilleurs clients de l'hôtel.

(c) Association des Amis de la faïence ancienne de Lunéville Saint-Clément.

Des membres de l'association se tenaient à la disposition des visiteurs, dont certains sont revenus avec une pièce de famille, demandant comment elle se situait dans le contexte de l'exposition. L'association a ainsi contribué à susciter un intérêt pour tel ou tel objet. La faïence prenait vie. Souvent, selon les témoignages recueillis auprès des visiteurs, l'objet était de nouveau utilisé par la famille, montré aux amis qui, à leur tour, cherchaient s'ils possédaient ce genre de pièces. Les membres de l'association ont découvert des objets méconnus, qui ont enrichi leur documentation photographique.

Une complicité s'est instaurée avec les propriétaires de pièces Rovina, réjouis de découvrir en vitrine des objets semblables aux leurs. Les membres de l'association ont eu parfois le sentiment d'« entrer dans la famille». On leur racontait qui avait offert l'objet ou comment les parents avaient acheté pièce par pièce un service complet, car «c'était trop cher pour acheter toutes les pièces d'un coup». Ces visiteurs se réappropriaient leurs pièces de faïence, qui trouvaient un nouveau sens. L'association a partagé avec eux une sorte d'héritage commun.

Quelques visiteurs s'interrogeaient sur le sort que leurs enfants réserveraient à ces pièces. La propriétaire d'un beau service à café, en or et platine, l'a confié à l'association pour son exposition permanente sur la faïencerie de Lunéville dans la grande salle que lui a cédée la municipalité. Ce service est devenu une part du patrimoine faïencier de l'association et est ainsi exposé à tous dans un espace muséal, lieu privilégié de la découverte du patrimoine. 


\section{De la patrimonialisation participative}

27 En écho à la définition donnée par Jean Davallon, qui écrit que « la patrimonialisation est le processus par lequel un collectif reconnaît le statut de patrimoine à des objets matériels ou immatériels, de sorte que le collectif se trouve devenir l'héritier de ceux qui les ont produits et qu'à ce titre il y a l'obligation de les garder afin de les transmettre $\aleph^{14}$, le cas présenté se caractérise par les allers-retours quasi permanents instaurés entre les membres de l'association, les experts extérieurs à l'association et les visiteurs. D'abord brefs, ces échanges deviennent peu à peu plus construits, se transformant à la fin du processus en une forme de complicité.

Une véritable implication naît chez les membres de l'association et les visiteurs. Les premiers mettent au service des amateurs curieux leur expertise dans le domaine de la faïence; les visiteurs compétents confortent le savoir des membres de l'association. Chacun apporte sa pierre à l'édifice. La connaissance se construit collectivement, au service de la préservation des objets. Au sein de l'association, un groupe de travail, constitué de spécialistes en histoire, en histoire de l'art et de collectionneurs de faïences, s'est constitué pour centraliser les informations et les analyser. Il recourt à des experts lorsqu'il n'est pas sûr de ses conclusions.

À tout moment, une personne extérieure à l'association peut apporter une information inédite, conférant une valeur et un éclairage nouveaux aux objets étudiés. Comme ce fut le cas pour les productions Rovina, l'objet est peu à peu intégré et reconnu comme faisant partie d'une histoire collective, méritant d'être conservé et présenté, après avis des membres du groupe.

La démarche ne s'arrête pas là car il est indispensable de formaliser les connaissances ainsi acquises. La manière de restituer ces connaissances est décidée d'un commun accord. Dans ce cas précis, un article a tout d'abord été écrit dans le journal de l'association, Le Réverbère. Mais cela s'est avéré insuffisant, les connaissances continuant à s'enrichir. L'intérêt de ces productions faïencières a paru tel que la volonté de les partager avec un plus large public s'est imposée.

31 Les recherches entreprises à propos de ces productions spinaliennes ont abouti à une réalisation temporaire, l'exposition, mais également à une forme pérenne, le catalogue. Ces travaux permettent de formaliser les connaissances acquises sur les objets et les techniques. Les chercheurs, les visiteurs partagent une connaissance commune élaborée à partir des apports de chacun, et chacun peut alors s'approprier ces objets. Pour que ces objets sortent de l'oubli, il faut les faire connaître et reconnaître par d'autres comme faisant partie d'un héritage commun. Et c'est pour ces raisons que l'association a pu recevoir en don un service à café : la donatrice $a$ vu là le meilleur moyen de préserver ces belles pièces et surtout, de partager avec d'autres ce qui lui appartenait, un héritage devenu bien de tous.

\section{Conclusion}

Il y a bien dans ce cas un changement de statut des objets ; avant en mains privées, ils deviennent propriété de tous. Chacun peut se reconnaitre dans ces objets, ils lui appartiennent. La production faïencière des ateliers Rovina a pris place dans le patrimoine lorrain, voire au-delà. Ce type de patrimonialisation relève d'une 
reconnaissance non institutionnelle, mais qui reste très forte car elle touche chaque individu et elle est synonyme de partage réel et de rencontres.

La démocratisation du patrimoine constitue depuis de longues décennies l'un des principaux objectifs de la politique culturelle institutionnelle. Elle prend ici une forme particulièrement intéressante d'appropriation par le public. Elle se développe dans une fructueuse coopération entre détenteurs d'objets, «professionnels » du patrimoine et amateurs éclairés. Cette étude de cas permet également de mettre en abyme un tel processus de patrimonialisation. Sous la Troisième République, à l'apogée de la production de la faïencerie de Lunéville, le thème de la démocratisation politique et sociale a beaucoup occupé les esprits, provoquant des débats passionnés entre ses partisans et ses adversaires. En 1913, l'historien et économiste Georges d'Avenel en fait un constat qui se veut scientifique et sans appel. Dans son ouvrage Le Nivellement des jouissances, il compare la vie quotidienne des Français de son temps avec celle des siècles passés. Il conclut ainsi son développement sur le service de table: "Pratiquement, les Français du $\mathrm{xx}^{\mathrm{e}}$ siècle mangent tous dans des assiettes à peu près pareilles de matière et d'aspect. Leur émail procure au prolétaire une jouissance positive de confortable (sic), tandis que les privilégiés n'ont plus à cet égard qu'un agrément assez artificiel de vanité15. » Aujourd'hui, il est possible de constater que ce sont la connaissance, l'usage et la constitution du patrimoine matériel que représentent désormais ces mêmes objets de la table qui, à leur tour, se démocratisent.

\section{NOTES}

1. - Voir le site : http://amisfaienceluneville.fr/ [consulté le 05/11/2019].

2. - ROCHE, Daniel. Histoire des choses banales. Naissance de la consommation dans les sociétés traditionnelles, XVII ${ }^{\mathrm{e}} \mathrm{XIX}{ }^{\mathrm{e}}$ siècle. Paris: Fayard, 1997; COQUERY, Natacha, COLLER, Ian et FLAMEIN, Richard. «Ce que les cultures matérielles peuvent apporter à l'historiographie de la Révolution française ». Annales historiques de la Révolution française, 2016/4, n 386, p. 125-144.

3. - RAMBOURG, Patrick. Histoire de la cuisine et de la gastronomie françaises. Paris : Perrin, 2009.

4. - Mémoires complets et authentiques du duc de Saint-Simon sur le siècle de Louis XIV et la Régence. Paris : Hachette, 1856, t. VII, p. 226-227, cités par FRANKLIN, Alfred. La Vie privée d'autrefois. Arts et métiers, modes, mœurs, usages des Parisiens du XII au XVIII'siècle d'après des documents originaux inédits. Paris : Plon-Nourrit, 1887-1902, t. VIII, Variétés gastronomiques, 1891.

5. - ROSEN, Jean. La Faïence en France du XIII ${ }^{e}$ au XIX siècle : technique et histoire. 2018, voir sur le site : https://halshs.archives-ouvertes.fr/halshs-01973891/file/Rosen\%202018.pdf; voir aussi, par exemple, l'histoire du développement de la faïence fine : MAIRE, Christian, SMITH, Helen, FAŸHALLÉ, Antoinette, TILLIARD, Laurence. Histoire de la faïence fine française, 1743-1843. Le triomphe des terres blanches. Cat. exp. Le Mans : Éditions de la Reinette, 2008.

6. - SAVOY, Bénédicte. Objets du désir. Désir d'objets. Histoire culturelle des patrimoines artistiques en Europe, $\mathrm{XVIII}^{e-\mathrm{XX}} \mathrm{X}^{e}$ siècle. Leçon inaugurale au Collège de France, 30 mars 2017. Paris : Collège de France/Fayard, 2017.

7. - GARNIER, Jacques et FOUCHET, Nelly. Faïences patriotiques. Paris : Varia, 1986-1992, fasc. 1-9. 
8. - «Lorsque Lunéville découvrait les arts de la table». Dossier de Lunéville - Les Cahiers du château, $\mathrm{n}^{\circ} 7,2011$.

9. - Voir le site : http://www.amisfaienceluneville.fr/ [consulté le 05/11/2019].

10. - LEVIEUGE, Guy et NOËL, Paulette. Rovina. Saga d'une famille de peintres sur faïnce et porcelaine. Lunéville : Les Amis de la Faïence ancienne de Lunéville Saint-Clément, 2017.

11. - Faïencerie située en Haute-Marne, très active au XVIII ${ }^{\mathrm{e}}$ siècle et réputée pour ses décors aux fleurs, oiseaux et chinois.

12. - René Fonck (1894-1953), pilote de chasse émérite pendant la Première Guerre mondiale, député des Vosges de 1919 à 1924.

13. - Gilbert Chasteloux (1908-1971) entre à 14 ans chez Schmitt-Rovina et apprend le métier de peintre. Aimée Résal, peintre en porcelaine et installée à son compte, soustraite certaines commandes des Rovina. Madeleine Xélot (1863-1953) et Marthe Balandier (1886-1974) sont deux cousines peintres céramistes installées à Mirecourt, qui ont dû travailler ponctuellement pour les ateliers Rovina.

14. - DAVALLON, Jean. À propos des régimes de patrimonialisation: enjeux et questions. Lisbonne : 2014, voir le site : https://halshs.archives-ouvertes.fr/halshs-01123906 [consulté le 05/11/2019]. 15. - AVENEL, Georges d'. Le Nivellement des jouissances. Paris : Flammarion, 1913, p. 84.

\section{RÉSUMÉS}

L'association des Amis de la faïence ancienne de Lunéville Saint-Clément, fondée en 1998, poursuit deux objectifs principaux: étudier et faire connaître le patrimoine faïencier des entreprises célèbres ou méconnues de Lunéville (Meurthe-et-Moselle) et sa région; partager les connaissances acquises avec les amateurs, étudiants, chercheurs et professionnels du patrimoine. La présente étude de cas, qui s'appuiera sur l'exemple de l'exposition consacrée en 2017 aux faïences de la famille Rovina, propose de rendre compte des formes de médiation mises actuellement en œuvre, notamment la démarche participative. L'association invite ainsi toute personne possédant des pièces de faïence à contribuer à une recherche documentaire et à des expositions.

The association of the friends of the old faience factory of Lunéville Saint-Clément was founded in 1998 and has two main aims: to study and promote the heritage of the faience produced by the famous (and less famous) faience factories of Lunéville, in the Meurthe-et-Moselle department, and to share information about this heritage with amateurs, students, researchers and heritage professionals. The present case study, based on an exhibition mounted in 2017 and devoted to the faience pieces of the Rovina family, will give an account of the different forms of mediation that the association uses, and in particular its participatory approach. The association invites owners of pieces of faience to make a contribution to the documentary research and to the exhibitions.

\section{INDEX}

Mots-clés : patrimonialisation, patrimoine, faïence, Lunéville, Rovina, information

Keywords : heritage identification, heritage, faience, Lunéville, Rovina, information 


\section{AUTEURS}

\section{JEANNINE GUENOT}

Historienne de l'art, documentaliste, Secrétaire des Amis de la faïence ancienne de Lunéville Saint-Clément et des Amis de l'histoire culturelle européenne guenot.jeannine@orange.fr

\section{DENIS SAILLARD}

Chercheur associé au Centre d'histoire culturelle des sociétés contemporaines (CHCSC), Université de Versailles Saint-Quentin (Paris-Saclay) denissaillard@sfr.fr 